Proceedings of the Institute of Mathematics and Mechanics,

National Academy of Sciences of Azerbaijan

Volume 45, Number 2, 2019, Pages 167-180

https://doi.org/10.29228/proc. 1

\title{
A NEW TECHNIQUE FOR FINDING EXACT SOLUTIONS OF NONLINEAR TIME-FRACTIONAL WAVE-LIKE EQUATIONS WITH VARIABLE COEFFICIENTS
}

\author{
ALI KHALOUTA AND ABDELOUAHAB KADEM
}

\begin{abstract}
In this paper, we give the exact solutions in terms of MittagLeffler functions for nonlinear time-fractional wave-like equations with variable coefficients by using a new technique, it is a combination of natural transform and variational iteration method called natural variational iteration method (NVIM). This technique enables us to overcome the difficulties arising in identifying the general Lagrange multiplier. The numerical results shows that this method is highly effective and is practically suitable for use in these equations. Three numerical examples are given to verify the accuracy and efficiency of the proposed technique.
\end{abstract}

\section{Introduction}

Fractional partial differential equations are widely used in interpretation and modeling of many of realism matters appear in applied mathematics and physics including fluid mechanics, electrical circuits, diffusion, damping laws, relaxation processes, mathematical biology, and so on. Therefore, to seek the solution of fractional partial differential equations is an important aspect of scientific research. Several mathematical methods including: Adomian decomposition method (ADM) [14], variational iteration method (VIM) [18] homotopy analysis method (HAM) [15] and homotopy perturbation method (HPM) [4], have been developed to obtain the exact and approximate analytic solutions.

Recently, a new option has appeared, includes the combination of Laplace transform, Sumudu transform or natural transform with the previously mentioned methods to facilitate and improve the resolution speed of fractional partial differential equations. Among wich are: Laplace homotopy analysis method [3], Laplace decomposition method [7], Laplace variational iteration method [17], homotopy perturbation Sumudu transform method [16], homotopy analysis Sumudu transform method [10], variational iteration Sumudu transform method [1], natural transform homotopy perturbation method [11], natural decomposition method [12], homotopy analysis natural transform method [13].

In this paper we will suggest a new technique to the search for exact solutions of nonlinear time-fractional wave-like equations with variable coefficients. This

2010 Mathematics Subject Classification. Primary 35R11, 26A33, Secondary 33E12, 35C05.

Key words and phrases. nonlinear time-fractional wave-like equations, Liouville-Caputo fractional derivative, Mittag-Leffler functions, natural transform, variational iteration method. 
technique is a combination of two powerful methods, natural transform and variational iteration method, called natural variational iteration method (NVIM).

Consider the following nonlinear time-fractional wave-like equations

$$
\begin{aligned}
D_{t}^{\alpha} v= & \sum_{i, j=1}^{n} F_{1 i j}(X, t, v) \frac{\partial^{k+m}}{\partial x_{i}^{k} \partial x_{j}^{m}} F_{2 i j}\left(v_{x_{i}}, v_{x_{j}}\right) \\
& +\sum_{i=1}^{n} G_{1 i}(X, t, v) \frac{\partial^{p}}{\partial x_{i}^{p}} G_{2 i}\left(v_{x_{i}}\right)+H(X, t, v)+S(X, t),
\end{aligned}
$$

with the initial conditions

$$
v(X, 0)=a_{0}(X), v_{t}(X, 0)=a_{1}(X),
$$

where $D_{t}^{\alpha}$ is the Liouville-Caputo fractional derivative operator of order $\alpha, 1<$ $\alpha \leq 2$.

Here $X=\left(x_{1}, x_{2}, \ldots, x_{n}\right), F_{1 i j}, G_{1 i} i, j \in\{1,2, \ldots, n\}$ are nonlinear functions of $X, t$ and $v, F_{2 i j}, G_{2 i} i, j \in\{1,2, \ldots, n\}$, are nonlinear functions of derivatives of $v$ with respect to $x_{i}$ and $x_{j} i, j \in\{1,2, \ldots, n\}$, respectively. Also $H, S$ are nonlinear functions and $k, m, p$ are integers.

These types of equations are of considerable significance in various fields of applied sciences, mathematical physics, nonlinear hydrodynamics, engineering physics, biophysics, human movement sciences, astrophysics and plasma physics. These equations describe the evolution of erratic motions of small particles that are immersed in fluids, fluctuations of the intensity of laser light, velocity distributions of fluid particles in turbulent flows.

The plan of this paper is structured as follows. In Section 2, we introduce some necessary definitions of the fractional calculus theory and natural transform, two theorems are introduced with their proofs related of the natural transform of fractional derivatives. In Section 3, we give a brief review of variational iteration method (VIM). In section 4, we introduce our results to solve the nonlinear timefractional wave-like equations (1.1) with the initial conditions (1.2) by the natural variational iteration method (NVIM). In section 5 , we present three numerical examples to show the efficiency of this technique and we present our obtained results graphically. All numerical results are obtained using Matlab (version R2016a). Finally, conclusions are drawn in the last section.

\section{Basic definitions}

In this section, we give some basic notions about fractional calculus, natural transform and natural transform of fractional derivatives which are used further in this paper.

2.1. Fractional calculus. We give some basic definitions and important properties of the fractional calculus theory as the Riemann-Liouville fractional integral and Liouville-Caputo fractional derivative (see[9]).

Definition 2.1. A real function $f(t), t>0$, is considered to be in the space $C_{\mu}$, $\mu \in \mathbb{R}$ if there exists a real number $p>\mu$, so that $f(t)=t^{p} h(t)$, where $h(t)$ $\in C\left(\left[0, \infty[)\right.\right.$, and it is said to be in the space $C_{\mu}^{n}$ if $f^{(n)} \in C_{\mu}, n \in \mathbb{N}$. 
Definition 2.2. The Riemann-Liouville fractional integral operator of order $\alpha \geq$ 0 of $f \in C_{\mu}, \mu \geq-1$ is defined as follows

$$
I^{\alpha} f(t)=\left\{\begin{array}{lc}
\frac{1}{\Gamma(\alpha)} \int_{0}^{t}(t-\xi)^{\alpha-1} f(\xi) d \xi, t>0, \alpha>0 \\
f(t), & \alpha=0
\end{array}\right.
$$

where $\Gamma($.$) is the well-known gamma function.$

Definition 2.3. The Liouville-Caputo fractional derivative of order $\alpha>0$ of $f \in C_{-1}^{n}, n \in \mathbb{N}$ is defined as follows

$$
D^{\alpha} f(t)=\left\{\begin{array}{l}
I^{n-\alpha} f^{(n)}(t), t>0, n-1<\alpha<n, \\
f^{(n)}(t), \quad \alpha=n .
\end{array}\right.
$$

For the Riemann-Liouville fractional integral and Liouville-Caputo fractional derivative, we have the following relation

$$
I^{\alpha} D^{\alpha} f(t)=f(t)-\sum_{k=0}^{n-1} f^{(k)}\left(0^{+}\right) \frac{t^{k}}{k !}, t>0 .
$$

Definition 2.4. (Partial derivatives of time-fractional order) Let $n$ to be the smallest integer that exceeds $\alpha$. The Liouville-Caputo time-fractional derivative operator of order $\alpha \in \mathbb{R}^{+}$is defined as follows

$$
D_{t}^{\alpha} v(x, t)=\left\{\begin{array}{lc}
\frac{1}{\Gamma(n-\alpha)} \int_{0}^{t}(t-\xi)^{n-\alpha-1} v^{(n)}(x, \xi) d \xi, & n-1<\alpha<n, \\
v^{(n)}(x, t), & \alpha=n .
\end{array}\right.
$$

Definition 2.5. The Mittag-Leffler function is defined as follows

$$
E_{\alpha}(z)=\sum_{n=0}^{\infty} \frac{z^{n}}{\Gamma(n \alpha+1)}, \alpha \in \mathbb{C}, \operatorname{Re}(\alpha)>0 .
$$

A further generalization of (2.5) is given in the form

$$
E_{\alpha, \beta}(z)=\sum_{n=0}^{\infty} \frac{z^{n}}{\Gamma(n \alpha+\beta)}, \alpha, \beta \in \mathbb{C}, \operatorname{Re}(\alpha)>0, \operatorname{Re}(\beta)>0 .
$$

For $\alpha=1, E_{\alpha}(z)$ reduces to $e^{z}$.

2.2. Natural transform. Recently, Fethi Bin Muhammed Belgacem et al. [2] introduced a new integral transform, called natural transform, which is applied to solve an ordinary and partial differential equations.

Definition 2.6. The natural transform is defined over the set of functions

$$
A=\left\{f(t) / \exists M, \tau_{1}, \tau_{2}>0,|f(t)|<M e^{\frac{|t|}{\tau_{j}}}, \text { ift } \in(-1)^{j} \times[0, \infty)\right\},
$$

by the following integral

$$
\mathcal{N}^{+}[f(t)]=R^{+}(s, u)=\frac{1}{u} \int_{0}^{+\infty} e^{-\frac{s t}{u}} f(t) d t, s, u \in(0, \infty) .
$$

Some basic properties of the natural transform are given as follows [2]. 
Property 1: The natural transform is a linear operator. That is, if $\lambda$ and $\mu$ are non-zero constants, then

$$
\mathcal{N}^{+}[\lambda f(t) \pm \mu g(t)]=\lambda \mathcal{N}^{+}[f(t)] \pm \mu \mathcal{N}^{+}[g(t)] .
$$

Property 2: If $f^{(n)}(t)$ is the $n^{t h}$ derivative of function $f(t)$ with respect to " $t$ " then its natural transform is given by

$$
\mathcal{N}^{+}\left[f^{(n)}(t)\right]=R_{n}^{+}(s, u)=\frac{s^{n}}{u^{n}} R^{+}(s, u)-\sum_{k=0}^{n-1} \frac{s^{n-(k+1)}}{u^{n-k}} f^{(k)}(0) .
$$

Property 3: (Convolution property) Suppose $F^{+}(s, u)$ and $G^{+}(s, u)$ are the natural transforms of $f(t)$ and $g(t)$, respectively, both defined in the set $A$. Then the natural transform of their convolution is given by

$$
\mathcal{N}^{+}[(f * g)(t)]=u F^{+}(s, u) G^{+}(s, u),
$$

where the convolution of two functions is defined by

$$
(f * g)(t)=\int_{0}^{t} f(\xi) g(t-\xi) d \xi=\int_{0}^{t} f(t-\xi) g(\xi) d \xi .
$$

\section{Property 4:}

$$
\begin{aligned}
\mathcal{N}^{+}[1] & =\frac{1}{s} \\
\mathcal{N}^{+}[t] & =\frac{u}{s^{2}}, \\
\mathcal{N}^{+}\left[\frac{t^{n-1}}{(n-1) !}\right] & =\frac{u^{n-1}}{s^{n}}, n=1,2, \ldots
\end{aligned}
$$

Property 5: If $\alpha>-1$, then the natural transform of $t^{\alpha}$ is given by

$$
\mathcal{N}^{+}\left[t^{\alpha}\right]=\Gamma(\alpha+1) \frac{u^{\alpha}}{s^{\alpha+1}} .
$$

\subsection{Natural transform of fractional derivatives.}

Theorem 2.1. If $R^{+}(s, u)$ is the natural transform of the function $f(t)$, then the natural transform of the Riemann-Liouville fractional integral of $f(t)$ of order $\alpha$, is given by

$$
\mathcal{N}^{+}\left[I^{\alpha} f(t)\right]=\frac{u^{\alpha}}{s^{\alpha}} R^{+}(s, u) .
$$

Proof. The Riemann-Liouville fractional integral for the function $f(t)$ as in (2.1), can be expressed as the convolution

$$
I^{\alpha} f(t)=\frac{1}{\Gamma(\alpha)} t^{\alpha-1} * f(t) .
$$

Applying the natural transform in the Eq. (2.9) and using properties (3) and (5), we have

$$
\begin{aligned}
\mathcal{N}^{+}\left[I^{\alpha} f(t)\right] & =\mathcal{N}^{+}\left[\frac{1}{\Gamma(\alpha)} t^{\alpha-1} * f(t)\right]=u \frac{1}{\Gamma(\alpha)} \mathcal{N}^{+}\left[t^{\alpha-1}\right] \mathcal{N}^{+}[f(t)] \\
& =u \frac{u^{\alpha-1}}{s^{\alpha}} R^{+}(s, u)=\frac{u^{\alpha}}{s^{\alpha}} R^{+}(s, u) .
\end{aligned}
$$

The proof is complete. 
Theorem 2.2. Let $n \in \mathbb{N}^{*}$ and $\alpha>0$ be such that $n-1<\alpha \leq n$ and $R^{+}(s, u)$ be the natural transform of the function $f(t)$, then the natural transform denoted by $R_{\alpha}^{+}(s, u)$ of the Liouville-Caputo fractional derivative of $f(t)$ of order $\alpha$, is given by

$$
\mathcal{N}^{+}\left[D^{\alpha} f(t)\right]=R_{\alpha}^{+}(s, u)=\frac{s^{\alpha}}{u^{\alpha}} R^{+}(s, u)-\sum_{k=0}^{n-1} \frac{s^{\alpha-(k+1)}}{u^{\alpha-k}}\left[D^{k} f(t)\right]_{t=0} .
$$

Proof. Let

$$
g(t)=f^{(n)}(t)
$$

then by the Definition 2.3 of the Liouville-Caputo fractional derivative, we obtain

$$
\begin{aligned}
D^{\alpha} f(t) & =\frac{1}{\Gamma(n-\alpha)} \int_{0}^{t}(t-\xi)^{n-\alpha-1} f^{(n)}(\xi) d \xi \\
& =\frac{1}{\Gamma(n-\alpha)} \int_{0}^{t}(t-\xi)^{n-\alpha-1} g(\xi) d \xi \\
& =I^{n-\alpha} g(t) .
\end{aligned}
$$

Applying the natural transform on both sides of (2.11) and using Eq. (2.8), we get

$$
\mathcal{N}^{+}\left[D^{\alpha} f(t)\right]=\mathcal{N}^{+}\left[I^{n-\alpha} g(t)\right]=\frac{u^{n-\alpha}}{s^{n-\alpha}} G^{+}(s, u) .
$$

Also, we have from the property (2)

$$
\begin{aligned}
\mathcal{N}^{+}[g(t)] & =\mathcal{N}^{+}\left[f^{(n)}(t)\right] \\
G^{+}(s, u) & =\frac{s^{n}}{u^{n}} R^{+}(s, u)-\sum_{k=0}^{n-1} \frac{s^{n-(k+1)}}{u^{n-k}}\left[f^{(k)}(t)\right]_{t=0} .
\end{aligned}
$$

Hence, (2.12) becomes

$$
\begin{aligned}
\mathcal{N}^{+}\left[D^{\alpha} f(t)\right] & =\frac{u^{n-\alpha}}{s^{n-\alpha}}\left(\frac{s^{n}}{u^{n}} R^{+}(s, u)-\sum_{k=0}^{n-1} \frac{s^{n-(k+1)}}{u^{n-k}} f^{(k)}(0)\right) \\
& =\frac{s^{\alpha}}{u^{\alpha}} R^{+}(s, u)-\sum_{k=0}^{n-1} \frac{s^{\alpha-(k+1)}}{u^{\alpha-k}}\left[D^{k} f(t)\right]_{t=0}=R_{\alpha}^{+}(s, u), \\
-1 & <n-1<\alpha \leq n .
\end{aligned}
$$

The proof is complete.

\section{Variational iteration method}

Idea of variational iteration method depends on the general Lagrange's multiplier method [6]. This method has a main feature, which is the solution of a mathematical problem with linearization assumption used as initial approximation or trial function. This approximation converges rapidly to an accurate solution. 
To illustrate the basic concepts of the VIM, we consider the following nonlinear differential equation

$$
L v(x, t)+N v(x, t)=g(x, t)
$$

where $L$ is a linear operator and $N$ is a nonlinear operator, and $g(x, t)$ is an inhomogeneous term.

According to the VIM [5], we can construct a correction functional as follows

$$
v_{n+1}(x, t)=v_{n}(x, t)+\int_{0}^{t} \lambda(\xi)[L v(x, \xi)+N \widetilde{v}(x, \xi)-g(x, \xi)] d \xi
$$

where $\lambda(\xi)$ is a general Lagrangian multiplier, which can be identified optimally via the variational theory and integration by parts. The subscript $n$ denotes the $n^{t h}$ order approximation, $\widetilde{v}_{n}$ is considered as a restricted variation (i.e. $\delta \widetilde{v}_{n}=0$ ).

So, we first determine the Lagrange multiplier $\lambda(\xi)$ that will be identified optimally via integration by parts. The successive approximations $v_{n+1}, n \geq 0$ of the solution $v(x, t)$ will be readily obtained upon using the obtained Lagrange multiplier and by using any selective function $v_{0}$. Consequently, the Solution

$$
v(x, t)=\lim _{n \longrightarrow \infty} v_{n}(x, t) .
$$

\section{Solution of nonlinear time-fractional wave-like equations by the natural variational iteration method (NVIM)}

Theorem 4.1. Consider the nonlinear time-fractional wave-like equations (1.1) with the initial conditions (1.2).

Then, by the NVIM the exact solution of Eqs. (1.1) and (1.2) is given as a limit of the successive approximations $\left\{v_{n}(X, t)\right\}_{n \geq 0}$, in other words,

$$
v(X, t)=\lim _{n \longrightarrow \infty} v_{n}(X, t) .
$$

Proof. In order to achieve our goal, we consider the following nonlinear timefractional wave-like equations (1.1) with the initial conditions (1.2).

First we define

$$
\begin{aligned}
N v & =\sum_{i, j=1}^{n} F_{1 i j}(X, t, v) \frac{\partial^{k+m}}{\partial x_{i}^{k} \partial x_{j}^{m}} F_{2 i j}\left(v_{x_{i}}, v_{x_{j}}\right), \\
M v & =\sum_{i=1}^{n} G_{1 i}(X, t, v) \frac{\partial^{p}}{\partial x_{i}^{p}} G_{2 i}\left(v_{x_{i}}\right), \\
K v & =H(X, t, v) .
\end{aligned}
$$

Eq. (1.1) is written in the form

$$
D_{t}^{\alpha} v(X, t)=N v(X, t)+M v(X, t)+K v(X, t)+S(X, t) .
$$

Applying the natural transform on both sides of (4.2) subject to the initial conditions (1.2) and using the Theorem 2.2, we get

$$
\begin{aligned}
\mathcal{N}^{+}[v(X, t)]= & \frac{1}{s} v(X, 0)+\frac{u}{s^{2}} v_{t}(X, 0)+\frac{u^{\alpha}}{s^{\alpha}} \mathcal{N}^{+}[S(X, t)] \\
& +\frac{u^{\alpha}}{s^{\alpha}} \mathcal{N}^{+}[N v(X, t)+M v(X, t)+K v(X, t)] .
\end{aligned}
$$


After that, let us take the inverse natural transform on both sides of (4.3) we have

$$
v(X, t)=L(X, t)+\mathcal{N}^{-1}\left(\frac{u^{\alpha}}{s^{\alpha}} \mathcal{N}^{+}[N v(X, t)+M v(X, t)+K v(X, t)]\right),
$$

where $L(X, t)$ is a term arising from the source term and the prescribed initial conditions.

Take the first partial derivative with respect to " $t$ " of Eq. (4.4) to obtain

$$
\begin{aligned}
0= & \frac{\partial}{\partial t} v(X, t)-\frac{\partial}{\partial t} L(X, t) \\
& -\frac{\partial}{\partial t} \mathcal{N}^{-1}\left(\frac{u^{\alpha}}{s^{\alpha}} \mathcal{N}^{+}[N v(X, t)+M v(X, t)+K v(X, t)]\right) .
\end{aligned}
$$

According to the variational iteration method, we can construct a correct functional as follows

$$
\begin{aligned}
& v_{n+1}(X, t)=v_{n}(X, t) \\
& -\int_{0}^{t}\left[\frac{\partial v_{n}}{\partial \xi}-\frac{\partial}{\partial \xi} \mathcal{N}^{-1}\left(\frac{u^{\alpha}}{s^{\alpha}} \mathcal{N}^{+}\left[N v_{n}+M v_{n}+K v_{n}\right]\right)-\frac{\partial L}{\partial \xi}\right] d \xi . \\
& \begin{aligned}
& \text { Or } \\
& v_{n+1}(X, t)= L(X, t) \\
&+\mathcal{N}^{-1}\left(\frac{u^{\alpha}}{s^{\alpha}} \mathcal{N}^{+}\left[N v_{n}(X, t)+M v_{n}(X, t)+K v_{n}(X, t)\right]\right) .
\end{aligned}
\end{aligned}
$$

Therefore, the exact solution of Eqs. (1.1) and (1.2) is given as a limit of the successive approximations $\left\{v_{n}(X, t)\right\}_{n \geq 0}$, in other words,

$$
v(X, t)=\lim _{n \rightarrow \infty} v_{n}(X, t) .
$$

This completes the proof.

\section{Illustrative Examples and Numerical Results}

In this section, we solve three numerical examples of nonlinear time-fractional wave-like equations with variable coefficients by using the NVIM.

Example 5.1. Consider the 2-dimensional nonlinear time-fractional wave-like equation with variable coefficients

$$
D_{t}^{\alpha} v=\frac{\partial^{2}}{\partial x \partial y}\left(v_{x x} v_{y y}\right)-\frac{\partial^{2}}{\partial x \partial y}\left(x y v_{x} v_{y}\right)-v, 1<\alpha \leq 2,
$$

with the initial conditions

$$
v(x, y, 0)=e^{x y}, v_{t}(x, y, 0)=e^{x y},
$$

where $D_{t}^{\alpha}$ is the Liouville-Caputo fractional derivative operator of order $\alpha, v$ is a function of $(x, y, t) \in \mathbb{R}^{2} \times \mathbb{R}^{+}$.

According to (4.7), we can construct the iteration formula as follows 


$$
\begin{aligned}
v_{n+1}(x, y, t)= & e^{x y}+t e^{x y}+\mathcal{N}^{-1}\left(\frac { u ^ { \alpha } } { s ^ { \alpha } } \mathcal { N } ^ { + } \left[\frac{\partial^{2}}{\partial x \partial y}\left(v_{n x x} v_{n y y}\right)\right.\right. \\
& \left.\left.-\frac{\partial^{2}}{\partial x \partial y}\left(x y v_{n x} v_{n y}\right)-v_{n}\right]\right)
\end{aligned}
$$

Using the iteration formula (5.3), we obtain

$$
\begin{aligned}
v_{0}= & (1+t) e^{x y} \\
v_{1}= & \left(1+t-\frac{t^{\alpha}}{\Gamma(\alpha+1)}-\frac{t^{\alpha+1}}{\Gamma(\alpha+2)}\right) e^{x y} \\
v_{2}= & \left(1+t-\frac{t^{\alpha}}{\Gamma(\alpha+1)}-\frac{t^{\alpha+1}}{\Gamma(\alpha+2)}+\frac{t^{2 \alpha}}{\Gamma(2 \alpha+1)}+\frac{t^{2 \alpha+1}}{\Gamma(2 \alpha+2)}\right) e^{x y} \\
& \ldots
\end{aligned}
$$

Then, the general term in successive approximation is given by

$$
v_{n}(x, y, t)=\sum_{k=0}^{n}\left(\frac{(-1)^{k} t^{k \alpha}}{\Gamma(k \alpha+1)}+\frac{(-1)^{k} t^{k \alpha+1}}{\Gamma(k \alpha+2)}\right) e^{x y} .
$$

So, the exact solution of Eqs. (5.1) and (5.2) in a closed form is given by

$$
\begin{aligned}
v(x, y, t) & =\lim _{n \rightarrow \infty} v_{n}(x, y, t)=\sum_{k=0}^{\infty}\left(\frac{(-1)^{k} t^{k \alpha}}{\Gamma(k \alpha+1)}+\frac{(-1)^{k} t^{k \alpha+1}}{\Gamma(k \alpha+2)}\right) e^{x y} \\
& =\left(E_{\alpha}\left(-t^{\alpha}\right)+t E_{\alpha, 2}\left(-t^{\alpha}\right)\right) e^{x y} .
\end{aligned}
$$

where $E_{\alpha}\left(-t^{\alpha}\right)$ and $E_{\alpha, 2}\left(-t^{\alpha}\right)$ are the Mittag-Leffler functions, defined by Eqs. (2.5) and (2.6).

For $\alpha=2$, then

$$
v(x, y, t)=\left(E_{2}\left(-t^{2}\right)+t E_{2,2}\left(-t^{2}\right)\right) e^{x y}=(\cos t+\sin t) e^{x y} .
$$

which is the same result as those obtained by the NIM and NHPM [8] for the same example. The surface and behavior of the solution for this example is graphically presented in Figures 1 and 2 for different fractional orders of $\alpha$.
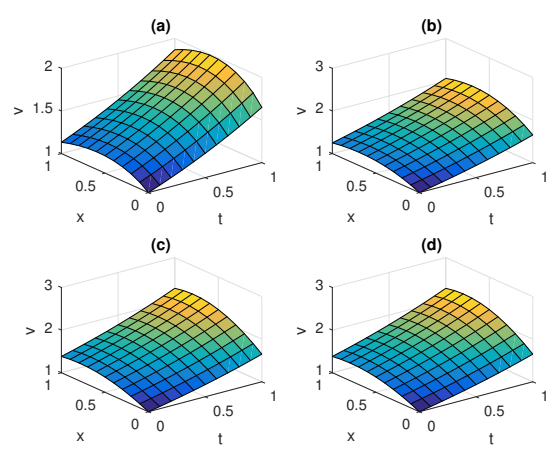

FiguRE 1. The surface graph of the $3^{\text {th }}$ order approximate solution by NVIM and the exact solution of Example 5.1 when $y=0.5$ : (a) $v$ when $\alpha=1.5$, (b) $v$ when $\alpha=1.75$, (c) $v$ when $\alpha=2$, and (d) $v$ is exact. 


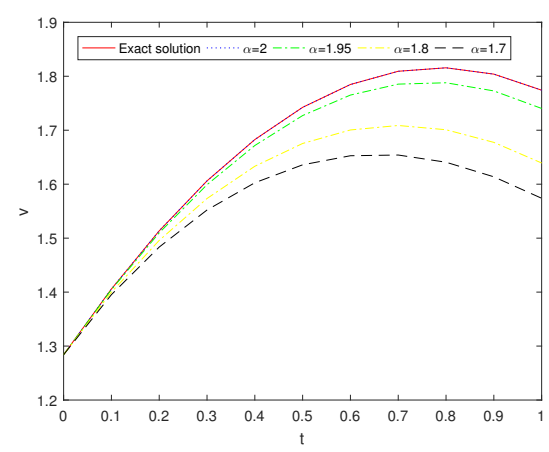

FIGURE 2. The behavior of the exact solution and the $3^{\text {th }}$ order approximate solution by NVIM of $v$ for different values of $\alpha$ for Example 5.1 when $x=y=0.5$.

\begin{tabular}{|c|c|c|c|c|c|c|}
\hline$t$ & $\alpha=1.7$ & $\alpha=1.8$ & $\alpha=1.95$ & $\alpha=2$ & exact solution & $\left|v_{\text {exact }}-v_{N V I M}\right|$ \\
\hline 0.1 & 1.3953 & 1.3999 & 1.4046 & 1.4058 & 1.4058 & $3.2196 \times 10^{-13}$ \\
0.3 & 1.5522 & 1.5735 & 1.5991 & 1.6061 & 1.6061 & $2.1569 \times 10^{-9}$ \\
0.5 & 1.6359 & 1.6755 & 1.7272 & 1.7424 & 1.7424 & $1.3095 \times 10^{-7}$ \\
0.7 & 1.6540 & 1.7088 & 1.7854 & 1.8093 & 1.8093 & $1.9680 \times 10^{-6}$ \\
0.9 & 1.6137 & 1.6775 & 1.7728 & 1.8040 & 1.8040 & $1.4947 \times 10^{-5}$ \\
\hline
\end{tabular}

TABLE 1. The numerical values of the $4^{\text {th }}$ order approximate solution and the exact solution for Example 5.1 when $x=y=0.5$.

Example 5.2. Consider the following nonlinear time-fractional wave-like equation with variable coefficients

$$
D_{t}^{\alpha} v=v^{2} \frac{\partial^{2}}{\partial x^{2}}\left(v_{x} v_{x x} v_{x x x}\right)+v_{x}^{2} \frac{\partial^{2}}{\partial x^{2}}\left(v_{x x}^{3}\right)-18 v^{5}+v, 1<\alpha \leq 2,
$$

with the initial conditions

$$
v(x, 0)=e^{x}, v_{t}(x, 0)=e^{x},
$$

where $D_{t}^{\alpha}$ is the Liouville-Caputo fractional derivative operator of order $\alpha$, and $v$ is a function of $(x, t) \in] 0,1\left[\times \mathbb{R}^{+}\right.$.

According to (4.7), we can construct the iteration formula as follows

$$
\begin{aligned}
v_{n+1}(x, t)= & e^{x}+t e^{x}+\mathcal{N}^{-1}\left(\frac { u ^ { \alpha } } { s ^ { \alpha } } \mathcal { N } ^ { + } \left[v_{n}^{2} \frac{\partial^{2}}{\partial x^{2}}\left(v_{n x} v_{n x x} v_{n x x x}\right)\right.\right. \\
& \left.\left.+v_{n x}^{2} \frac{\partial^{2}}{\partial x^{2}}\left(v_{n x x}^{3}\right)-18 v_{n}^{5}+v_{n}\right]\right) .
\end{aligned}
$$


Using the iteration formula (5.6), we obtain

$$
\begin{aligned}
v_{0}= & (1+t) e^{x} \\
v_{1}= & \left(1+t+\frac{t^{\alpha}}{\Gamma(\alpha+1)}+\frac{t^{\alpha+1}}{\Gamma(\alpha+2)}\right) e^{x}, \\
v_{2}= & \left(1+t+\frac{t^{\alpha}}{\Gamma(\alpha+1)}+\frac{t^{\alpha+1}}{\Gamma(\alpha+2)}+\frac{t^{2 \alpha}}{\Gamma(2 \alpha+1)}+\frac{t^{2 \alpha+1}}{\Gamma(2 \alpha+2)}\right) e^{x},
\end{aligned}
$$

and so on.

Then, the general term in successive approximation is given by

$$
v_{n}(x, t)=\sum_{k=0}^{n}\left(\frac{t^{k \alpha}}{\Gamma(k \alpha+1)}+\frac{t^{k \alpha+1}}{\Gamma(k \alpha+2)}\right) e^{x} .
$$

So, the exact solution of Eqs. (5.4) and (5.5) in a closed form is given by

$$
\begin{aligned}
v(x, t) & =\lim _{n \rightarrow \infty} v_{n}(x, t)=\sum_{k=0}^{\infty}\left(\frac{t^{k \alpha}}{\Gamma(k \alpha+1)}+\frac{t^{k \alpha+1}}{\Gamma(k \alpha+2)}\right) e^{x} \\
& =\left(E_{\alpha}\left(t^{\alpha}\right)+t E_{\alpha, 2}\left(t^{\alpha}\right)\right) e^{x},
\end{aligned}
$$

where $E_{\alpha}\left(t^{\alpha}\right)$ and $E_{\alpha, 2}\left(t^{\alpha}\right)$ are the Mittag-Leffler functions, defined by Eqs. (2.5) and (2.6).

For $\alpha=2$, then

$$
v(x, t)=\left(E_{2}\left(t^{2}\right)+t E_{2,2}\left(t^{2}\right)\right) e^{x}=e^{x+t} .
$$

which is the same result as those obtained by the NIM and NHPM [8] for the same example. The surface and behavior of the solution for this example is graphically presented in Figures 3 and 4 for different fractional orders of $\alpha$.
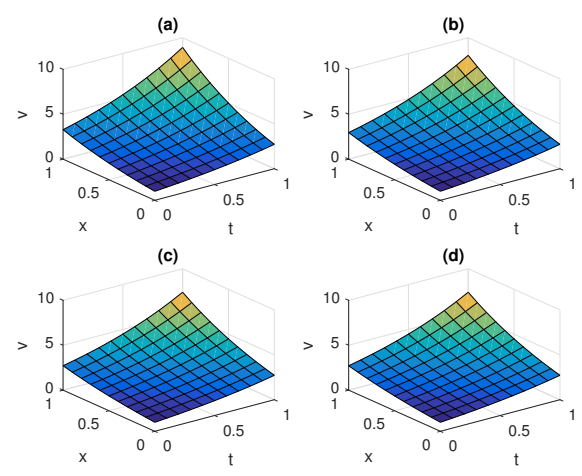

Figure 3 . The surface graph of the $3^{\text {th }}$ order approximate solution by NVIM and the exact solution of Example 5.2: (a) $v$ when $\alpha=1.5$, (b) $v$ when $\alpha=1.75$, (c) $v$ when $\alpha=2$, and (d) $v$ is exact. 


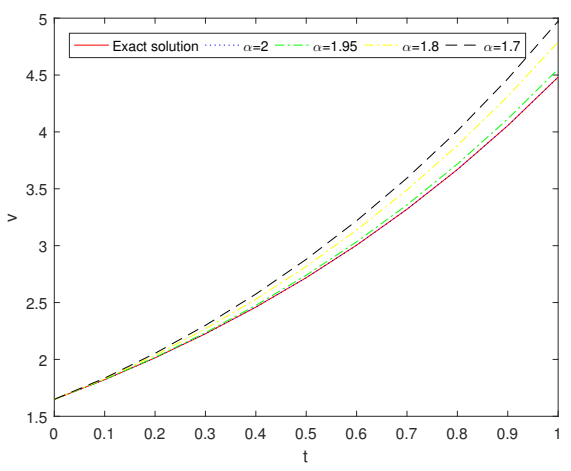

FIGURE 4 . The behavior of the exact solution and the $3^{\text {th }}$ order approximate solution by NVIM of $v$ for different values of $\alpha$ for Example 5.2 when $x=0.5$.

\begin{tabular}{|c|c|c|c|c|c|c|}
\hline$t$ & $\alpha=1.7$ & $\alpha=1.8$ & $\alpha=1.95$ & $\alpha=2$ & exact solution & $\left|v_{\text {exact }}-v_{N V I M}\right|$ \\
\hline 0.1 & 1.8357 & 1.8298 & 1.8236 & 1.8221 & 1.8221 & $4.1350 \times 10^{-13}$ \\
0.3 & 2.2994 & 2.2697 & 2.2350 & 2.2255 & 2.2255 & $2.7750 \times 10^{-9}$ \\
0.5 & 2.8800 & 2.8174 & 2.7402 & 2.7183 & 2.7183 & $1.6907 \times 10^{-7}$ \\
0.7 & 3.5940 & 3.4901 & 3.3585 & 3.3201 & 3.3201 & $2.5543 \times 10^{-6}$ \\
0.9 & 4.4670 & 4.3129 & 4.1140 & 4.0552 & 4.0552 & $1.9535 \times 10^{-5}$ \\
\hline
\end{tabular}

TABLE 2. The numerical values of the $4^{\text {th }}$ order approximate solution and the exact solution for Example 5.2 when $x=0.5$.

Example 5.3. Consider the following one-dimensional nonlinear time-fractional wave-like equation with variable coefficients

$$
D_{t}^{\alpha} v=x^{2} \frac{\partial}{\partial x}\left(v_{x} v_{x x}\right)-x^{2}\left(v_{x x}\right)^{2}-v, 1<\alpha \leq 2,
$$

with the initial conditions

$$
v(x, 0)=0, v_{t}(x, 0)=x^{2},
$$

where $D_{t}^{\alpha}$ is the Liouville-Caputo fractional derivative operator of order $\alpha$, and $v$ is a function of $(x, t)(x, t) \in] 0,1\left[\times \mathbb{R}^{+}\right.$.

According to (4.7), we can construct the iteration formula as follows

$$
v_{n+1}(x, t)=t x^{2}+\mathcal{N}^{-1}\left(\frac{u^{\alpha}}{s^{\alpha}} \mathcal{N}^{+}\left[x^{2} \frac{\partial}{\partial x}\left(v_{n x} v_{n x x}\right)-x^{2}\left(v_{n x x}\right)^{2}-v_{n}\right]\right) .
$$

Using the iteration formula (5.9), we obtain

$$
v_{0}=t x^{2}, v_{1}=\left(t-\frac{t^{\alpha+1}}{\Gamma(\alpha+2)}\right) x^{2}, v_{2}=\left(t-\frac{t^{\alpha+1}}{\Gamma(\alpha+2)}+\frac{t^{2 \alpha+1}}{\Gamma(2 \alpha+2)}\right) x^{2}, \ldots
$$

and so on. 
Then, the general term in successive approximation is given by

$$
v_{n}(x, t)=x^{2}\left(\sum_{k=0}^{n} \frac{(-1)^{k} t^{k \alpha+1}}{\Gamma(k \alpha+2)}\right) .
$$

So, the exact solution of Eqs. (5.7) and (5.8) in a closed form is given by

$$
v(x, t)=\lim _{n \rightarrow \infty} v_{n}(x, t)=x^{2}\left(\sum_{i=0}^{\infty} \frac{(-1)^{k} t^{k \alpha+1}}{\Gamma(k \alpha+2)}\right)=x^{2}\left(t E_{\alpha, 2}\left(-t^{\alpha}\right)\right),
$$

where $E_{\alpha, 2}\left(-t^{\alpha}\right)$ is the Mittag-Leffler function, defined by Eq. (2.6).

For $\alpha=2$, then

$$
v(x, t)=x^{2}\left(t E_{2,2}\left(-t^{2}\right)\right)=x^{2} \sin t .
$$

which is the same result as those obtained by the NIM and NHPM [8] for the same example. The surface and behavior of the solution for this example is graphically presented in Figures 5 and 6 for different fractional orders of $\alpha$.
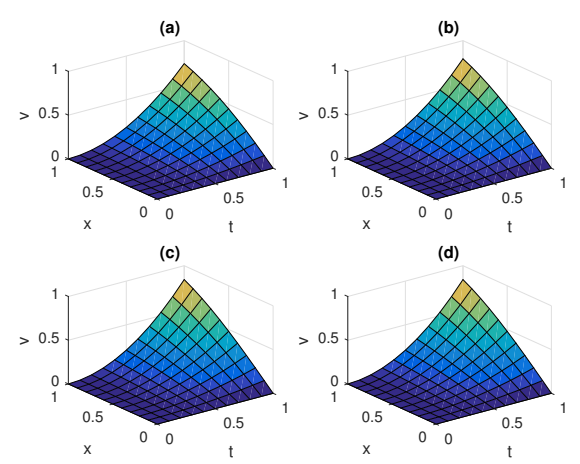

Figure 5. The surface graph of the $3^{\text {th }}$ order approximate solution by NVIM and the exact solution of Example 5.3: (a) $v$ when $\alpha=1.5$, (b) $v$ when $\alpha=1.75$, (c) $v$ when $\alpha=2$, and (d) $v$ is exact.

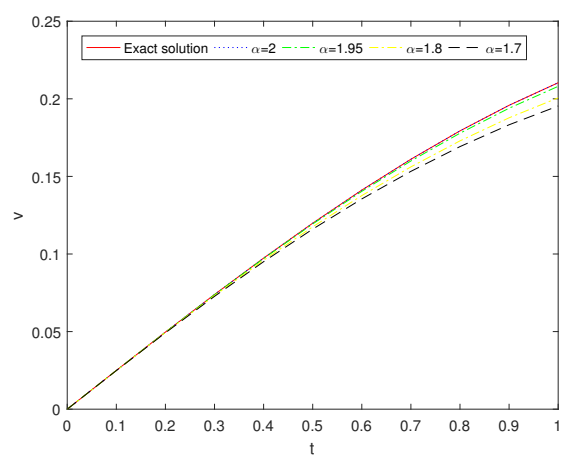

Figure 6 . The behavior of the exact solution and the $3^{\text {th }}$ order approximate solution by NVIM of $v$ for different values of $\alpha$ for Example 5.3 when $x=0.5$. 


\begin{tabular}{|c|c|c|c|c|c|c|}
\hline$t$ & $\alpha=1.7$ & $\alpha=1.8$ & $\alpha=1.95$ & $\alpha=2$ & exact solution & $\left|v_{\text {exact }}-v_{N V I M}\right|$ \\
\hline 0.1 & 0.02488 & 0.02492 & 0.02495 & 0.02496 & 0.02496 & $6.8887 \times 10^{-16}$ \\
0.3 & 0.07271 & 0.07319 & 0.07374 & 0.07388 & 0.07388 & $1.3549 \times 10^{-11}$ \\
0.5 & 0.11604 & 0.11752 & 0.11934 & 0.11986 & 0.11986 & $1.3425 \times 10^{-9}$ \\
0.7 & 0.15325 & 0.15615 & 0.15994 & 0.16105 & 0.16105 & $2.7677 \times 10^{-8}$ \\
0.9 & 0.18327 & 0.18777 & 0.19394 & 0.19583 & 0.19583 & $2.6495 \times 10^{-7}$ \\
\hline
\end{tabular}

TABLE 3 . The numerical values of the $4^{\text {th }}$ approximate solution and the exact solution for Example 5.3 when $x=0.5$.

\section{Conclution}

In this paper, a new technique called natural variational iteration method (NVIM) is presented for finding the exact solutions of the nonlinear time-fractional wave-like equations with variable coefficients. This technique is used to overcome the complexity of identifying the general Lagrange multiplier. When compared with the existing published techniques, it is easy to notice that the new technique has many advantages. It is straightforward, easy to understand, and fast, requiring much less computations to perform a limited number of steps of the simple procedure that can be applied to find the exact solution of a wide range of types of fractional partial differential equations. Furthermore, there is no need for using linearization or restrictive assumptions when employing this new method. The method was tested on three numerical examples on different situations. Numerical results obtained by the technique confirm the easily, accurately and efficiency of the technique, and therefore, can be widely applied in others fractional partial differential equations.

Acknowledgements. The authors are very grateful to both the Editor-inChief Professor Misir Mardanov, Managing Editor Professor Vugar Ismailov and the referees for carefully reading the paper and for their important remarks and suggestions in order to improve it.

\section{References}

[1] A. S. Abedl-Rady, S. Z. Rida, A. A. M. Arafa and H. R. Abedl-Rahim, Variational Iteration Sumudu Transform Method for Solving Fractional Nonlinear Gas Dynamics Equation, Int. J. Res. Stu. Sci. Eng. Tech. 1 (2014), 82-90.

[2] F. B. M. Belgacem and R. Silambarasan, Theory of natural transform, Math. Eng. Sci. Aerosp. 3 (2012), no.1, 105-135.

[3] V. G. Gupta, P. Kumar, Approximate solutions of fractional linear and nonlinear differential equations using Laplace homotopy analysis method, Int. J. Nonlinear Sci. 19 (2015), no.2, 113-120.

[4] M. Hamdi Cherif, K. Belghaba and D. Ziane, Homotopy perturbation method for solving the fractional Fisher's equation, Int. J. Anal. Appl. 10 (2016), no.1, 9-16.

[5] J. H. He, Variational iteration method-some recent results and new interpretations, J. Comput. Appl. Math. 207 (2007), 3-17.

[6] M. Inokuti, H. Sekine and T. Mura, General use of the Lagrange multiplier in nonlinear mathematical physics. In: Nemat-Nassed S, editor. Variational Method in the Mechanics of Solids, Pergamon Press, Oxford, 1978, 156-162. 
[7] H. Jafari, C. M. Khalique and M. Nazari, Application of the Laplace decomposition method for solving linear and nonlinear fractional diffusion-wave equations, Appl. Math. Lett. 24 (2011), 1799-1805.

[8] A. Khalouta and A. Kadem, Comparison of New Iterative Method and Natural Homotopy Perturbation Method for Solving Nonlinear Time-Fractional Wave-Like Equations with Variable Coefficients, Nonlinear Dyn. Syst. Theory. 19(1-SI) (2019), $160-169$.

[9] A. Kilbas, H. M. Srivastava, and J. J. Trujillo, Theory and Application of Fractional Differential equations, Elsevier, North-Holland, 2006.

[10] R. P. Kumar and H. M. Kumar, Homotopy analysis Sumudu transform method for time-fractional third order dispersive partial differential equation, Adv. Comput. Math. 43 (2017), 365-383.

[11] S. Maitama, A Hybrid Natural Transform Homotopy Perturbation Method for Solving Fractional Partial Differential Equations, Int. J. Differ. Equ. (2016), 1-7.

[12] M. S. Rawashdeh and S. Maitama, Solving Coupled System of Nonliear PDEs Using the Naturel Decomposition Method, Int. J. of Pure. Appl. Math. 92 (2014), no.5, $757-776$.

[13] S. Z. Rida, A. A. A. Arafa, A. S. Abedl-Rady and H. R. Abdl-Rahim, Homotopy Analysis Natural Transform Method For Solving Fractional Physique Models, Int. J. Pure Appl. Math. 117 (2017), no.1, 19-32.

[14] L. Song and W. Wang, A new improved Adomian decomposition method and its application to fractional differential equations, Appl. Math. Mod. 37 (2013), 15901598 .

[15] K. Vishal, S. Kumar and S. Das, Application of homotopy analysis method for fractional Swift Hohenberg equation Revisited, Appl. Math. Model. 36 (2012), 36303637.

[16] E. A. Yousif and S. H. M. Hamed, Solution of Nonlinear Fractional Differential Equations Using the Homotopy Perturbation Sumudu Transform Method, Appl. Math. Sci. 8 (2014), no.44, 2195-2210.

[17] A. M. Yang, J. Li, H . M. Srivastava, G-N, Xie and X-J Yang, Local Fractional Laplace Variational Iteration Method for Solving Linear Partial Differential Equations with Local Fractional Derivative, Discrete Dyn. Nat. Soc. (2014), 1-8.

[18] Y. Zhang, Time-Fractional Generalized Equal Width Wave Equations: Formulation and Solution via Variational Methods, Nonlinear Dyn. Syst. Theory. 14 (2014), no. $12,410-425$.

\section{Ali Khalouta}

Laboratory of Fundamental and Numerical Mathematics, Department of Mathematics, Faculty of Sciences, Ferhat Abbas Sétif University 1, 19000 Sétif, Algeria.

E-mail address: nadjibkh@yahoo.fr

\section{Abdelouahab Kadem}

Laboratory of Fundamental and Numerical Mathematics, Department of Mathematics, Faculty of Sciences, Ferhat Abbas Sétif University 1, 19000 Sétif, Algeria.

E-mail address: abdelouahabk@yahoo.fr

Received: February 7, 2019; Revised: April 21, 2019; Accepted: April 22, 2019 